Pacific

Journal of

Mathematics

ON IMAGINARY QUADRATIC NUMBER FIELDS WITH 2-CLASS GROUP OF RANK 4 AND INFINITE 2-CLASS FIELD TOWER

ElLiot Benjamin 


\title{
ON IMAGINARY QUADRATIC NUMBER FIELDS WITH 2-CLASS GROUP OF RANK 4 AND INFINITE 2-CLASS FIELD TOWER
}

\author{
Elliot Benjamin
}

Let $k$ be an imaginary quadratic number field with $C_{k, 2}$, the 2-Sylow subgroup of its ideal class group $C_{k}$, of rank 4 . We show that $k$ has infinite 2-class field tower for particular families of fields $k$, according to the 4-rank of $C_{k}$, the Kronecker symbols of the primes dividing the discriminant $\Delta_{k}$ of $k$, and the number of negative prime discriminants dividing $\Delta_{k}$. In particular we show that if the 4-rank of $C_{k}$ is greater than or equal to 2 and exactly one negative prime discriminant divides $\Delta_{k}$, then $k$ has infinite 2-class field tower.

\section{Introduction.}

Let $k$ denote an algebraic number field and $C_{k, 2}$ denote its 2-class group, i.e., the 2-Sylow subgroup of the ideal class group $C_{k}$ (in the wide sense) of $k$; denote by $k_{1}$ the Hilbert 2-class field of $k$. Let $k_{n}$ (for $n$ a nonnegative integer) be defined inductively as $k_{0}=k$ and $k_{n+1}=\left(k_{n}\right)_{1}$. Then $k_{0} \subseteq k_{1} \subseteq$ $k_{2} \subseteq \ldots \subseteq k_{n} \subseteq \ldots$ is called the 2-class field tower of $k$. If $n$ is the minimal integer such that $k_{n}=k_{n+1}$, then $n$ is called the length of the tower. If no such $n$ exists, then the tower is said to be of infinite length.

In 1964, Golod and Shafarevich (cf. [4]) established for the first time the existence of infinite $p$-class field towers, for $p$ prime. In the case $p=2$, their criterion (as refined by Gaschütz and Vinberg [10]) can be stated in the following way, where $E_{k, 2}$ denotes the unit group of $k$ mod its squares, $E_{k} / E_{k}^{2}$ : If rank $C_{k, 2} \geq 2+2\left(\operatorname{rank} E_{k, 2}+1\right)^{1 / 2}$ then $k$ has infinite 2-class field tower. We shall refer to the above inequality as the Golod-Shafarevich inequality. We immediately see that for $k$ imaginary with rank $C_{k, 2} \geq 5$, or $k$ real with rank $C_{k, 2} \geq 6$, the Golod-Shafarevich inequality is satisfied and $k$ thereby has infinite 2-class field tower. It is well-known that for $k$ imaginary with rank $C_{k, 2}=2$ or 3 , the 2-class field tower of $k$ may be finite or infinite, and that if rank $C_{k, 2}=1$ then the 2-class field tower of $k$ is finite and has length 1 (cf. [3], [6], [14], [17], [20]). It has been conjectured that for $k$ imaginary with rank $C_{k, 2}=4, k$ has infinite 2-class field tower (cf. [17], [18]). 
A partial result in this direction, as proved by Hajir, is that if $k$ is an imaginary quadratic number field such that $C_{k, 2}$ contains a subgroup isomorphic to $Z_{4} \times Z_{4} \times Z_{4}$, then $k$ has infinite 2-class field tower (cf. [6], [7]). We extend this result to particular fields $k$ with rank $C_{k, 2}=4$ and 4-rank of $C_{k}$ less than 3. Denoting the discriminant of $k$ by $\Delta_{k}$, our fields $k$ are classified according to the 4-rank of $C_{k}$, the Kronecker symbols $(p / q)$ of the primes dividing $\Delta_{k}$, and the number of negative prime discriminants dividing $\Delta_{k}$. We demonstrate that if the 4-rank of $C_{k}$ is greater than or equal to 2 and exactly one negative prime discriminant divides $\Delta_{k}$, then $k$ has infinite 2-class field tower.

\section{Preliminaries.}

Our initial results are directly related to the following inequality (cf. [6], $[7])$ :

Proposition 1. Let $F$ be a totally real field of degree $n, E$ a totally complex quadratic extension of $F$, and $t$ the number of prime ideals of $F$ which ramify in $E$. If $t \geq 3+2 \sqrt{n+1}$ then the 2 -class field tower of $E$ is infinite.

We will also need to utilize the well-known ambiguous class number formula, where for a cyclic extension $K / F$ an ambiguous ideal class is an ideal class of $K$ that remains invariant under the action of $\mathrm{Gal}(K / F)$. We denote the subgroup of ambiguous ideal classes by $\operatorname{Am}(K / F)$ and its Sylow 2-subgroup by $\operatorname{Am}_{2}(K / F)$. We state the following two propositions: (cf. [12], [15]).

Proposition 2. Let $K / F$ be a cyclic extension of prime degree $p$. Then $|\operatorname{Am}(K / F)|=h(F) \cdot p^{t-1} /(E: H)$ where $t$ is the number of (finite or infinite) primes of $F$ which are ramified in $K / F, E=E_{F}$ is the unit group of $F$, $H=E \cap N_{K / F} K^{x}$ is the subgroup of units which are norms of elements of $K^{x}$, and $K^{x}$ is the multiplicative group of $K$.

Proposition 3. Let $K / F$ be a quadratic extension of an algebraic number field where $h(F)$ is odd. Then $\left|\operatorname{Am}_{2}(K / F)\right|=2^{e}$ where $e$ is the 2-rank of $C_{K}$.

\section{Results.}

We begin by obtaining some conditions on the Kronecker symbols of the primes dividing $\Delta_{k}$, directly related to Proposition 1 , to insure that an imaginary quadratic number field $k$ with rank $C_{k, 2}=4$ has infinite 2-class field tower.

Lemma 1. Let $k$ be an imaginary quadratic number field such that rank $C_{k, 2}=4$. If for some prime $p_{j} \equiv 1 \bmod 4$, or $p_{j}=2$ in which case we further assume that 8 is a fundamental discriminant dividing $\Delta_{k}$, we have 
$\left(\frac{p_{j}}{p_{k}}\right)=\left(\frac{p_{j}}{p_{l}}\right)=\left(\frac{p_{j}}{p_{m}}\right)=1, p_{j}, p_{k}, p_{l}, p_{m}$ distinct primes, $p_{j} p_{k} p_{l} p_{m} \mid \Delta_{k}$, then $k$ has infinite 2 -class field tower.

Proof. We proceed in a similar way to Hajir in [6]. Let $F=Q\left(\sqrt{p_{j}}\right)$, $E=k\left(\sqrt{p_{j}}\right)$. We see that $E$ is a $C M$ field with maximal field subfield $F$ such that either 7 or 8 primes ramify from $F$ to $E$. Since $7 \geq 3+2 \sqrt{2+1}$, it follows from Proposition 1 that $E$ has infinite 2-class field tower. Since $E$ is an umramified quadratic extension of $k, k$ has infinite 2-class field tower as well.

We utilize the following notational convenience: If $d_{j}$ is a negative prime discriminant we let $p_{j}$ denote the prime dividing $d_{j}$ if $d_{j} \neq-4$, and $p_{j}=1$ if $d_{j}=-4$.

Lemma 2. Let $k$ be an imaginary quadratic number field such that rank $C_{k, 2}=n, n \geq 1$. Let $L=Q\left(\sqrt{d_{j}}\right)$ and $F=k\left(\sqrt{d_{j}}\right)$, where $d_{j}$ is a negative prime discriminant, $p_{j} \mid \Delta_{k}$. Then exactly $2 n$ prime ideals in $L$ are ramified in $F$ if and only if $\left(\frac{-p_{j}}{p_{i}}\right)=1, i \neq j$, for all primes $p_{i} \mid \Delta_{k}, 1 \leq i \leq n+1$.

Proof. Assume $\left(\frac{-p_{j}}{p_{i}}\right)=1, i \neq j$, for all primes $p_{i} \mid \Delta_{k}, 1 \leq i \leq n+1$. It follows that there are exactly $n$ primes $p_{i}$ dividing $\Delta_{k}$ that split in $L=$ $Q\left(\sqrt{d_{j}}\right)$. Since $F=k\left(\sqrt{d_{j}}\right)$ is an unramified quadratic extension of $k$, these $n$ primes $p_{i}$ each have ramification index 2 in $F$. We therefore can conclude that each of these primes $p_{i}$ must ramify from $L$ to $F$. There are no other prime ideals that ramify from $L$ to $F$, since if there were a prime ideal $P_{m}$ in $L$ that ramifies in $F$ such that $P_{m} \cap Q=p_{m} \neq p_{i}, 1 \leq i \leq n+1$, it would imply that $p_{m}$ ramifies in $F$. But $p_{m}$ does not divide $\Delta_{k}$ unless $p_{m}=p_{j}$, and $F$ is an unramified quadratic extension of $k$. Since $p_{j}$ has ramification index 2 in $F$, we therefore conclude that exactly $2 n$ prime ideals in $L$ are ramified in $F$. The converse is proved in a similar way and is left to the reader.

We note that in our proof of Lemma 1 we were able to utilize the full strength of Proposition 1 by requiring only 7 primes to ramify from $F$ to $E$, whereas Hajir, in his original proof that if the 4-rank of $C_{k}$ is greater than or equal to 3 then $k$ has infinite 2-class field tower (cf. [6]), assumed that $p_{i} \equiv 1 \bmod 4,\left(\frac{p_{j}}{p_{i}}\right)=1, j \neq i$, and therefore 8 primes ramified from $F$ to E.

We illustrate Hajir's method of proof of the above result in the case where a negative prime discriminant $d_{j}$ divides $\Delta_{k},\left(\frac{-p_{j}}{p_{i}}\right)=1, j \neq i$, as follows (cf. [7]):

Lemma 3. Let $k$ be an imaginary quadratic number field such that rank $C_{k, 2}=4$. Assume there exists a negative prime discriminant $d_{j}, d_{j} \mid \Delta_{k}$, 
such that $,\left(\frac{-p_{j}}{p_{i}}\right)=1, j \neq i$, for all primes $p_{i} \mid \Delta_{k}, 1 \leq i \leq 5$. Then $k$ has infinite 2-class field tower.

Proof. Let $L=Q\left(\sqrt{-p_{j}}\right)$ and $F=k\left(\sqrt{-p_{j}}\right)$. By Lemma 2 we see that exactly 8 prime ideals in $L$ are ramified in $F$. By Proposition 2 and Proposition 3, it follows that rank $C_{F, 2} \geq 6$. Since rank $E_{F, 2}=2$, we obtain the Golod-Shafarevich inequality: $\operatorname{rank} C_{F, 2} \geq 6 \geq 2+2 \sqrt{2+1}$ and therefore $F$ has infinite 2 -class field tower. Since $F$ is an unramified quadratic extension of $k, k$ has infinite 2-class field tower as well.

We state the following corollaries of Lemma 3:

Corollary 1. Let $k$ be an imaginary quadratic number field such that rank $C_{k, 2}=4$, exactly one negative prime discriminant divides $\Delta_{k}$, and $\Delta_{k} \equiv$ $4 \bmod 8$. Then $k$ has infinite 2-class field tower.

Proof. Since $k$ has exactly one negative prime discriminant and $\Delta_{k} \equiv$ $4 \bmod 8$, all the odd primes dividing $\Delta_{k}$ are congruent to $1 \bmod 4$. We therefore have $\left(\frac{-1}{p_{i}}\right)=1$ for all odd primes $p_{i} \mid \Delta_{k}$ and our result follows immediately from Lemma 3 .

Corollary 2. Let $k$ be an imaginary quadratic number field, rank $C_{k, 2}=4$, such that five negative prime discriminants divide $\Delta_{k}$. Then the following fields $k$ have infinite 2 -class field tower, where $q_{i}, 1 \leq i \leq 5$, is a prime congruent to $3 \bmod 4$ :

$$
\begin{aligned}
Q\left(\sqrt{-q_{1} q_{2} q_{3} q_{4} q_{5}}\right), \quad\left(\frac{-q_{i}}{q_{j}}\right)=\left(\frac{-q_{i}}{q_{k}}\right)= & \left(\frac{-q_{i}}{q_{l}}\right)=\left(\frac{-q_{i}}{q_{m}}\right)=1, \\
& \{i, j, k, l, m\}=\{1,2,3,4,5\} \\
Q\left(\sqrt{-q_{1} q_{2} q_{3} q_{4}}\right), \quad\left(\frac{-q_{i}}{q_{j}}\right)=\left(\frac{-q_{i}}{q_{k}}\right)= & \left(\frac{-q_{i}}{q_{l}}\right)=\left(\frac{-q_{i}}{2}\right)=1, \\
& \{i, j, k, l\}=\{1,2,3,4\} .
\end{aligned}
$$

Proof. It is immediate by applying Lemma 3 to each field $k$ that we have infinite 2-class field tower. We note that these are all the possible fields satisfying the assumptions of our corollary for which we are able to apply Lemma 3.

For the cases when exactly one negative prime discriminant divides $\Delta_{k}$ where $\Delta_{k} \not \equiv 4 \bmod 8$, and exactly three negative prime discriminants divide $\Delta_{k}$, we utilize the following lemma:

Lemma 4. Let $k$ be an imaginary quadratic number field such that rank $C_{k, 2}=4$, at least two of the prime discriminants dividing $\Delta_{k}$ are positive, and $\left(\frac{p_{1}}{p_{3}}\right)=\left(\frac{p_{2}}{p_{3}}\right)=1$ where $p_{1}$ and $p_{2}$ are distinct primes dividing positive 
prime discriminants dividing $\Delta_{k}$, and $p_{3}$ is a prime dividing a positive or negative prime discriminant dividing $\Delta_{k}, p_{1} \neq p_{3} \neq p_{2}$. Then $k$ has infinite 2-class field tower.

Proof. By the assumptions of our lemma, we can write $k=Q\left(\sqrt{-p_{1} p_{2} p_{3} p_{4} p_{5}}\right)$ or $k=Q\left(\sqrt{-p_{1} p_{2} p_{3} p_{4}}\right)$ where $p_{1} \equiv p_{2} \equiv 1 \bmod 4$, or $p_{1}=2$ and $p_{2} \equiv$ $1 \bmod 4$. By Martinet (cf. [17], Proposition 5) we see immediately that $k$ has infinite 2-class field tower.

We now let $k=Q\left(\sqrt{-p_{1} p_{2} p_{3} p_{4} p_{5}}\right)$ where $\Delta_{k} \not \equiv 4 \bmod 8$ and exactly one negative prime discriminant divides $\Delta_{k}$. We define a Kronecker symbol configuration of $k$ to be a complete list of Kronecker symbols $\left(\frac{p_{i}}{p_{j}}\right), i \leq j$, $l \leq i \leq 5, l \leq j \leq 5$. We denote a Kronecker symbol configuration by listing all the Kronecker symbols $\left(\frac{p_{i}}{p_{j}}\right)$ as above with $\left(\frac{p_{i}}{p_{j}}\right)=1$ (respectively -1 ), where the remaining Kronecker symbols $\left(\frac{p_{i}}{p_{j}}\right), i<j$, are assumed to be -1 (respectively 1 ).

In Table 1 we utilize the Rédei \& Reichardt conditions [19] to list all possible Kronecker symbol configurations (without loss of generality) according to the 4-rank of $C_{k}$.

Table 1.

\begin{tabular}{|c|l|}
\hline 4-rank of $C_{k}$ & possible Kronecker symbol configurations \\
\hline 4 & all Kronecker symbols equal 1 \\
\hline 3 & $\left(\frac{p_{1}}{p_{2}}\right)=\left(\frac{p_{1}}{p_{3}}\right)=\left(\frac{p_{1}}{p_{4}}\right)=\left(\frac{p_{1}}{p_{5}}\right)=1$ \\
& $\left(\frac{p_{1}}{p_{2}}\right)=-1$ \\
\hline 2 & $\left(\frac{p_{1}}{p_{2}}\right)=\left(\frac{p_{1}}{p_{3}}\right)=\left(\frac{p_{2}}{p_{3}}\right)=1$ \\
& $\left(\frac{p_{1}}{p_{2}}\right)=\left(\frac{p_{3}}{p_{4}}\right)=\left(\frac{p_{1}}{p_{5}}\right)=\left(\frac{p_{2}}{p_{5}}\right)=1$ \\
& $\left(\frac{p_{1}}{p_{2}}\right)=\left(\frac{p_{1}}{p_{3}}\right)=\left(\frac{p_{1}}{p_{4}}\right)=\left(\frac{p_{1}}{p_{5}}\right)=\left(\frac{p_{2}}{p_{5}}\right)=1$ \\
& $\left(\frac{p_{1}}{p_{2}}\right)=\left(\frac{p_{3}}{p_{4}}\right)=-1$ \\
& $\left(\frac{p_{1}}{p_{2}}\right)=\left(\frac{p_{1}}{p_{3}}\right)=-1$ \\
& $\left(\frac{p_{1}}{p_{2}}\right)=\left(\frac{p_{1}}{p_{3}}\right)=\left(\frac{p_{2}}{p_{3}}\right)=-1$ \\
& $\left(\frac{p_{1}}{p_{2}}\right)=\left(\frac{p_{3}}{p_{5}}\right)=\left(\frac{p_{1}}{p_{3}}\right)=\left(\frac{p_{1}}{p_{5}}\right)=\left(\frac{p_{2}}{p_{3}}\right)=1$ \\
& $\left(\frac{p_{1}}{p_{2}}\right)=\left(\frac{p_{4}}{p_{5}}\right)=\left(\frac{p_{1}}{p_{3}}\right)=\left(\frac{p_{1}}{p_{5}}\right)=\left(\frac{p_{2}}{p_{3}}\right)=1$ \\
\hline
\end{tabular}




\begin{tabular}{|l|l|}
\hline 4-rank of $C_{k}$ & possible Kronecker symbol configurations \\
\hline 1 & $\left(\frac{p_{1}}{p_{2}}\right)=\left(\frac{p_{1}}{p_{3}}\right)=\left(\frac{p_{1}}{p_{4}}\right)=\left(\frac{p_{2}}{p_{3}}\right)=1$ \\
$\left(\frac{p_{1}}{p_{2}}\right)=\left(\frac{p_{1}}{p_{3}}\right)=\left(\frac{p_{1}}{p_{4}}\right)=\left(\frac{p_{2}}{p_{3}}\right)=-1$ \\
$\left(\frac{p_{1}}{p_{2}}\right)=\left(\frac{p_{1}}{p_{3}}\right)=\left(\frac{p_{1}}{p_{4}}\right)=-1$ \\
$\left(\frac{p_{1}}{p_{2}}\right)=\left(\frac{p_{1}}{p_{3}}\right)=\left(\frac{p_{2}}{p_{4}}\right)=-1$ \\
$\left(\frac{p_{1}}{p_{2}}\right)=\left(\frac{p_{1}}{p_{3}}\right)=1$ \\
\hline all Kronecker symbols equal -1 \\
& $\left(\frac{p_{1}}{p_{2}}\right)=1$ \\
$\left(\frac{p_{1}}{p_{2}}\right)=\left(\frac{p_{3}}{p_{4}}\right)=1$ \\
$\left(\frac{p_{1}}{p_{2}}\right)=\left(\frac{p_{1}}{p_{3}}\right)=\left(\frac{p_{2}}{p_{4}}\right)=1$ \\
$\left(\frac{p_{1}}{p_{2}}\right)=\left(\frac{p_{3}}{p_{4}}\right)=\left(\frac{p_{1}}{p_{5}}\right)=\left(\frac{p_{2}}{p_{3}}\right)=1$ \\
$\left(\frac{p_{1}}{p_{2}}\right)=\left(\frac{p_{3}}{p_{4}}\right)=\left(\frac{p_{1}}{p_{5}}\right)=\left(\frac{p_{2}}{p_{3}}\right)=\left(\frac{p_{4}}{p_{5}}\right)=1$ \\
$\left(\frac{p_{1}}{p_{2}}\right)=\left(\frac{p_{1}}{p_{3}}\right)=\left(\frac{p_{1}}{p_{4}}\right)=\left(\frac{p_{2}}{p_{5}}\right)=\left(\frac{p_{3}}{p_{5}}\right)=1$ \\
$\left(\frac{p_{1}}{p_{2}}\right)=\left(\frac{p_{3}}{p_{4}}\right)=\left(\frac{p_{1}}{p_{5}}\right)=1$ \\
$\left(\frac{p_{1}}{p_{2}}\right)=\left(\frac{p_{1}}{p_{3}}\right)=\left(\frac{p_{1}}{p_{4}}\right)=1$ \\
$\left(\frac{p_{1}}{p_{2}}\right)=\left(\frac{p_{3}}{p_{4}}\right)=\left(\frac{p_{1}}{p_{3}}\right)=\left(\frac{p_{1}}{p_{5}}\right)=1$ \\
$\left(\frac{p_{1}}{p_{2}}\right)=\left(\frac{p_{3}}{p_{4}}\right)=\left(\frac{p_{1}}{p_{3}}\right)=\left(\frac{p_{1}}{p_{5}}\right)=\left(\frac{p_{2}}{p_{3}}\right)=1$ \\
$\left(\frac{p_{1}}{p_{2}}\right)=\left(\frac{p_{3}}{p_{4}}\right)=\left(\frac{p_{1}}{p_{5}}\right)=-1$ \\
$\left(\frac{p_{1}}{p_{2}}\right)=\left(\frac{p_{3}}{p_{4}}\right)=\left(\frac{p_{1}}{p_{5}}\right)=\left(\frac{p_{2}}{p_{3}}\right)=-1$ \\
$\left(\frac{p_{1}}{p_{2}}\right)=\left(\frac{p_{3}}{p_{4}}\right)=\left(\frac{p_{1}}{p_{3}}\right)=\left(\frac{p_{1}}{p_{5}}\right)=-1$ \\
$\left(\frac{p_{1}}{p_{2}}\right)=\left(\frac{p_{3}}{p_{4}}\right)=\left(\frac{p_{1}}{p_{5}}\right)=\left(\frac{p_{2}}{p_{5}}\right)=1$ \\
$\left(\frac{p_{1}}{p_{2}}\right)=\left(\frac{p_{1}}{p_{3}}\right)=\left(\frac{p_{1}}{p_{4}}\right)=\left(\frac{p_{1}}{p_{5}}\right)=-1$ \\
\hline 0
\end{tabular}

We are now able to state the following theorem:

Theorem 1. Let $k$ be an imaginary quadratic number field with $\operatorname{rank} C_{k, 2}=$ $4, \Delta_{k} \not \equiv 4 \bmod 8$, and exactly one negative prime discriminant dividing $\Delta_{k}$. Then the following fields $k$ have infinite 2-class field tower, where $\{i, j, k, l, m\}=\{1,2,3,4,5\}$ :

(A) 4-rank of $C_{k}$ equal to 2,3 or 4 
(B) 4-rank of $C_{k}$ equal to 1 and Kronecker symbol configuration of $k$ not $\left(\frac{p_{i}}{p_{j}}\right)=\left(\frac{p_{i}}{p_{k}}\right)=1$ where either $p_{j}$ or $p_{k}$ is the prime dividing the negative prime discriminant dividing $d_{k}$

(C) 4-rank of $C_{k}$ equal to 0 (i.e., $C_{k, 2}$ elementary) and Kronecker symbol configuration of $k$ not one of the following types:

- all Kronecker symbols equal -1

- $\left(\frac{p_{i}}{p_{j}}\right)=1$

- $\left(\frac{p_{i}}{p_{j}}\right)=\left(\frac{p_{k}}{p_{l}}\right)=1$

- $\left(\frac{p_{i}}{p_{j}}\right)=\left(\frac{p_{k}}{p_{l}}\right)=\left(\frac{p_{i}}{p_{m}}\right)=1$ where either $p_{j}$ or $p_{m}$ is the prime dividing the negative prime discriminant dividing $d_{k}$.

Proof. For case (A) with 4-rank of $C_{k}$ equal to 3 or 4 the result has been established by Hajir (cf. [6], [7]). For case (A) with 4-rank of $C_{k}$ equal to 2 , and cases (B) and (C), we apply Lemma 4 to our fields listed in Table 1 to establish our result.

From Table 1 we see that there are 32 possible Kronecker symbol configurations when exactly one negative prime discriminant divides $\Delta_{k}, \Delta_{k} \not \equiv 4$ mod 8. From Theorem 1 we find that for 27 of these Kronecker symbol configurations, $k$ has infinite 2-class field tower. The unknown cases can be summarized by means of the 4-rank of $C_{k}$ as follows:

Table 2.

\begin{tabular}{|c|c|}
\hline 4-rank of $C_{k}$ & $\begin{array}{l}\text { number of Kronecker symbol } \\
\text { configurations where 2-class } \\
\text { field tower of } k \text { may be finite }\end{array}$ \\
\hline 4 & 0 \\
\hline 3 & 0 \\
\hline 2 & 0 \\
\hline 1 & 1 \\
\hline 0 & 4 \\
\hline
\end{tabular}

Remark 1. For the case when exactly three negative prime discriminants divide $\Delta_{k}$, one can again utilize the Rédei \& Reichardt conditions and Lemma 4 to obtain fields with infinite 2-class field tower. We note that in this case the Kronecker symbol configuration $\left(\frac{p_{1}}{p_{2}}\right)=\left(\frac{p_{1}}{p_{3}}\right)=\left(\frac{p_{1}}{p_{4}}\right)=1$ may not satisfy the requirements of Lemma 4; however, Lemma 1 may be used when $p_{1} \not \equiv 1 \bmod 4$, or when $p_{1}=2$ if 8 is a fundamental discriminant dividing $\Delta_{k}$. For the case when five negative prime discriminants divide $\Delta_{k}$, one can utilize the Rédei \& Reichardt conditions and Corollary 2 to obtain 
fields with infinite 2-class field tower. In a follow-up paper we will demonstrate that our techniques allow us to conclude that $k$ has infinite 2-class field tower for imaginary quadratic number fields $k$ when rank $C_{k, 2}=4, C_{k}$ has 4-rank equal to 2 , and either five negative prime discriminants divide $\Delta_{k}$ or $\Delta_{k} \not \equiv 4 \bmod 8$ (cf. [1]).

\section{Examples.}

From Lemma 1, Corollary 2, and Theorem 1 we immediately obtain that the following fields $k$ have infinite 2 -class field tower. We list our fields according to the 4-rank of $C_{k}$.

$C_{k, 2}$ elementary: $k=Q(\sqrt{-61,620})=Q(\sqrt{-3 \cdot 5 \cdot 13 \cdot 79})$

$$
\begin{aligned}
& k=Q(\sqrt{-120,180})=Q(\sqrt{-5 \cdot 13 \cdot 17 \cdot 29}) \\
& k=Q(\sqrt{-122,655})=Q(\sqrt{-3 \cdot 5 \cdot 13 \cdot 17 \cdot 37}) \\
& k=Q(\sqrt{-212,135})=Q(\sqrt{-5 \cdot 7 \cdot 11 \cdot 19 \cdot 29}) \\
& k=Q(\sqrt{-256,360})=Q(\sqrt{-2 \cdot 5 \cdot 13 \cdot 17 \cdot 29}) \\
& k=Q(\sqrt{-430,360})=Q(\sqrt{-2 \cdot 5 \cdot 7 \cdot 29 \cdot 53}) \\
& k=Q(\sqrt{-440,115})=Q(\sqrt{-3 \cdot 5 \cdot 13 \cdot 37 \cdot 61}) \\
& k=Q(\sqrt{-850,135})=Q(\sqrt{-5 \cdot 11 \cdot 13 \cdot 29 \cdot 41}) \\
& k=Q(\sqrt{-2,035,240})=Q(\sqrt{-2 \cdot 5 \cdot 17 \cdot 41 \cdot 73}) \\
& k=Q(\sqrt{-5,863,655})=Q(\sqrt{-5 \cdot 7 \cdot 29 \cdot 53 \cdot 109})
\end{aligned}
$$

$4-\operatorname{rank}$ of $C_{k}=1: \quad k=Q(\sqrt{-184,008})=Q(\sqrt{-2 \cdot 3 \cdot 11 \cdot 17 \cdot 41})$

$$
\begin{aligned}
& k=Q(\sqrt{-531,867})=Q(\sqrt{-3 \cdot 7 \cdot 19 \cdot 31 \cdot 43}) \\
& k=Q(\sqrt{-2,657,415})=Q(\sqrt{-3 \cdot 5 \cdot 29 \cdot 41 \cdot 149}) \\
& k=Q(\sqrt{-6,425,679})=Q(\sqrt{-3 \cdot 13 \cdot 37 \cdot 61 \cdot 73})
\end{aligned}
$$

$4-\operatorname{rank}$ of $C_{k}=2: \quad k=Q(\sqrt{-3,989,095})=Q(\sqrt{-5 \cdot 11 \cdot 29 \cdot 41 \cdot 61})$ 
Remark 2. Since the fields satisfying the conditions of Lemmas 1, 3, and 4 possess an unramified quadratic extension which satisfy the Golod-Shafarevich inequality, (cf. [19] in regard to Lemmas 1 and 4) it follows from Theorem 6 of Hajir (cf. [5]) that the rank of the 2-class groups of these fields tend to infinity.

In conclusion, we see that the conjecture concerning the 2-class field tower of $k$ being infinite holds in a number of particular fields $k$ when the 4-rank of $C_{k}$ is equal to 0,1 , or 2 , and always holds when the 4-rank of $C_{k}$ is greater than or equal to 3 . Our techniques allow us to obtain families of fields $k$ with 4-rank of $C_{k}$ equal to 0,1 , or 2 and k having infinite 2-class field tower, as well as the rank of the 2-class groups of the fields in the tower of $k$ tending to infinity. However, the complete resolution of the conjecture concerning all imaginary quadratic number fields $k$ with rank $C_{k, 2}=4$ is still a very open question.

Acknowledgement. I would like to express my gratitude to Chip Snyder for a number of helpful suggestions throughout this paper.

\section{References}

[1] E. Benjamin, On a question of Martinet concerrning the 2-class field tower of imaginary quadratic number fields, Annales des sciences; Mathematiques du Quebec (to appear).

[2] E. Benjamin and C.J. Parry, Refined lower bounds on the 2-class number of the Hilbert 2-class field of imaginary quadratic number fields with elementary 2-class group of rank 3, J. of Number Theory, 76 (1999), 167-177, MR 2000b:11125, Zbl 929.11047.

[3] E. Benjamin, C. Snyder and F. Lemmermeyer, Imaginary quadratic fields with cyclic $\mathrm{Cl}_{2}\left(K^{\mid}\right)$, J. of Number Theory, 67 (1997), 229-245, MR 99a:11126, Zbl 919.11074, Zbl 919.11074.

[4] E. Golod and I. Shafarevich, On (the) class field towers, Izv. Akad. Nauk SSSR, 28 (1964), 261-272 (in Russian); English translation: Amer. Math. Soc. Transl., 48 (1965), 91-102, Amer. Math. Soc., Providence, R.I., MR 28 \#5056, Zbl 148.28101.

[5] F. Hajir, On the growth of p-class groups in p-class field towers, J. of Algebra, 188 (1997), 256-271, MR 98a:11151, Zbl 879.11069.

[6] _ On a theorem of Koch, Pacific J. Math., 176(1) (1996), 15-18, MR 97k:11161, Zbl 879.11066.

[7] _ Correction to 'On a theorem of Koch', Pacific J. Math., 196(2) (2000), 507508, CMP 1800589.

[8] K. Iwasawa, A note on the group of units of an algebraic number field, J. Math. Pures Appl., 35 (1956), 189-192, MR 17,946h, Zbl 071.26504.

[9] G. Janusz, Algebraic Number Fields, Academic Press, New York, 1973, MR 51 \#3110, Zbl 307.12001.

[10] H. Koch, Zum satz von Golod-Schafarewitsch, Math. Nachr., 42 (1968), 321-333, MR 45 \#380, Zbl 191.33702. 
[11] S. Lang, Algebraic Number Theory, Springer Verlag, New York, 1994, MR 95f:11085, Zbl 811.11001.

[12] _ Cyclotomic Fields I, II, Springer Verlag, New York (1978, 1980), MR 58 \#5578, Zbl 395.12005; MR 81i:12004, Zbl 435.12001.

[13] F. Lemmermeyer, Class field towers, preprint.

[14] _ On 2-class field towers of some imaginary quadratic number fields, Abh. Math. Sem. Univ. Hamburg, 67 (1997), 205-214, MR 98k:11168, Zbl 919.11075.

[15] _ Explizite konstruktion von Hilbert-klassenkörpern, Diss. Univ. Heidelberg (1995).

[16] S. Louboutin, Norme relative de l'unité fondamentale et 2-rang du groupe des classes d'idéaux de certains corps biquadratiques, Acta Arith., 58 (1991), 273-288, MR 93a:11090, Zbl 726.11067.

[17] J. Martinet, Tours de corps de classes et estimations de discriminant, Inv. Math., 44 (1978), 65-73, MR 57 \#275, Zbl 369.12007.

[18] _ Discriminant de corps de nombres, Journées Arithmétiques 1980 (JV Armitage, ed.), Cambridge Univ. Press, 1987, 151-193, MR 84g:12009, Zbl 491.12005.

[19] L. Rédei and H. Reichardt, Die anzahl der durch 4 teilbaren invarienten der klassengruppe eines beliebigen quadratischen zahlkorpers, J. Reine Angew. Math., 170 (1966), 69-74.

[20] B. Schmithals, Konstruktion imaginärquadratischer körper mit unendlichem klassenkörperturm, Arch. Math., 34 (1980), 307-312, MR 82f:12017, Zbl 448.12008.

Received December 9, 1999 and revised April 19, 2000.

Deparment of Mathematics

Unity College

Unity, Maine 04988-9502

E-mail address: benjamin@mint.net 